\title{
SPOLM2019
}

XIXSIMPÓSIO DE PESQUISA OPERACIONAL E LOGÍSTICA DA MARINHA

\section{LOCALIZAÇÃO E ROTEAMENTO EM DOIS NÍVEIS NA DISTRIBUIÇÃO DE PRODUTOS EM ÁREAS URBANAS}

\author{
Vitória Pureza \\ Departamento de Engenharia de Produção - Universidade Federal de São Carlos \\ Rodovia Washington Luiz, km 235 \\ vpureza@dep.ufscar.br \\ Juan Sebastián Poveda Gulfo \\ Departamento de Engenharia de Produção - Universidade Federal de São Carlos \\ Rodovia Washington Luiz, km 235 \\ juanpovedag@gmail.com
}

\begin{abstract}
RESUMO
O problema de localização-roteamento em dois níveis com janelas de tempo e múltiplos entregadores é um complexo problema logístico que surge em atividades reais de distribuição de grandes volumes de produtos, notadamente em cidades de médio e grande porte. Devido às dificuldades de circulação e estacionamento veicular, a distribuição é realizada em dois níveis: no primeiro nível, os veículos se deslocam entre agrupamentos de clientes próximos, e no segundo nível, o motorista e seus ajudantes se deslocam a pé entre os clientes de um mesmo agrupamento a partir do local de parada do veículo. Neste trabalho é descrito um modelo de programação matemática para o problema. São também reportados resultados com exemplos gerados a partir de instâncias de um problema correlato e com base em dados reais de uma empresa distribuidora brasileira.
\end{abstract}

Palavra-chave: Localização; Roteamento multi-nível; Múltiplos entregadores; Logística urbana.

\begin{abstract}
The two-echelon location-routing problem with time windows and multiple deliverymen is a complex logistic problem that appears in real-life distribution of large volume of products, particularly in medium to large-sized cities. Because of driving and parking difficulties, the distribution is carried out on two levels; each vehicle travels between groups (clusters) of customers close to each other, and for each cluster, the delivery of the products is performed by the driver and possibly some helpers traveling with the driver and who visit the customers on foot from the vehicle stop location. This work describes a mathematical programming model for the problem. It is also reported results with examples generated from a correlated problem instances and based on real data from a Brazilian distributor.
\end{abstract}

Keywords: Location; Multilevel Routing; Multiple Deliverymen; Urban Logistics. 


\section{INTRODUÇÃO}

A presente pesquisa endereça as operações de distribuição de grandes volumes de produtos a clientes em regiões centrais de cidades de médio a grande porte. A distribuição é feita por uma frota de veículos baseada em um depósito, e devido às dificuldades de circulação e de estacionamento inerentes a tais regiões, cada veículo se desloca entre agrupamentos de clientes próximos entre si (clusters), enquanto a entrega dos produtos aos clientes de cada cluster é realizada a pé pelo motorista (e possivelmente alguns ajudantes) a partir do local de parada do veículo (Figura 1). Exemplos típicos de empresas que fazem uso desta prática são as distribuidoras de refrigerantes, cervejas e cigarros, cujos clientes em sua maioria consistem de pontos de venda concentrados em "bolsões" de áreas comerciais.

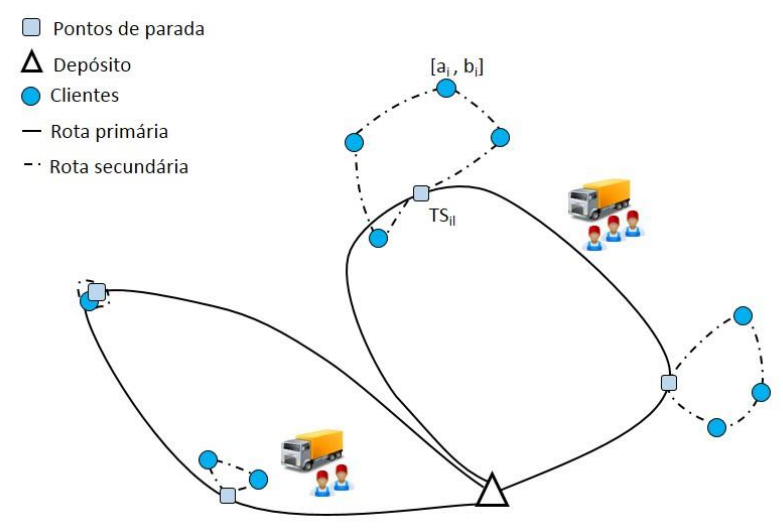

Figura 1: Rotas típicas de distribuição em áreas centrais de cidades de médio a grande porte.

O problema pode ser visto como uma variante do clássico problema de roteamento de veículos com janelas de tempo. As janelas de tempo são geralmente impostas por estabelecimentos em que o instante de entrega é um fator importante para suas próprias operações, tais como restaurantes ou lanchonetes que requerem que os produtos solicitados sejam entregues uma ou duas horas antes do horário do almoço.

O uso de ajudantes (múltiplos entregadores), por sua vez, tem como objetivo a redução do tempo de serviço nos clusters, permitindo o aumento do número de clientes atendidos durante a jornada de trabalho diária. Este objetivo é especialmente importante para o setor de refrigerantes e cervejas. Como se trata de um mercado com grande demanda e altamente competitivo, empresas produtoras procuram entregar os pedidos diários no mesmo dia, o que frequentemente excede a jornada de trabalho dos motoristas e ajudantes, e pode implicar em custos adicionais em horas extras bastante relevantes (SOUZA NETO; PUREZA, 2016).

Historicamente, o artigo de Pureza, Morabito e Reimann (2012) introduz o conceito com a proposição do Problema de Roteamento de Veículos com Janelas de Tempo e Múltiplos Entregadores (Vehicle Routing Problem with Time Windows and Multiple Deliverymen - VRPTWMD), e apresenta um modelo de programação matemática, um algoritmo de Busca Tabu e um algoritmo de Otimização por Colônia de Formigas para sua resolução. O problema se caracteriza por utilizar uma frota homogênea e ilimitada de veículos, disposta em apenas um depósito de onde os veículos partem e para onde devem retornar até o fim da jornada diária, e um número limitado de entregadores. Outro artigo seminal no tema é o de Ferreira e Pureza (2012). As autoras abordam o Problema de 
Roteamento de Veículos com Múltiplos Entregadores (Vehicle Routing Problem with Multiple Deliverymen - VRPMD), considerando uma frota de veículos homogênea, porém limitada, disponibilidade ilimitada de entregadores e permitindo o atendimento parcial dos clusters. Esta variação foi adotada por incluir elementos mais realistas ao problema, com base em um estudo de caso realizado. As autoras apresentam um modelo de programação matemática e propõem uma adaptação do algoritmo de economias de Clarke e Wright (1964) para sua resolução.

Outros métodos de solução para o VRPTWMD são apresentados em Senarclens De Grancy e Reimann (2014a), especificamente, um algoritmo de Otimização por Colônia de Formigas (ACO) e um algoritmo GRASP. Munari e Morabito (2016), por sua vez, apresentam um algoritmo branch-price-and-cut utilizado para resolver uma formulação de particionamento de conjuntos introduzida pelos autores, enquanto Álvarez Díaz (2016) propõe uma abordagem heurística e uma abordagem híbrida. A abordagem heurística utiliza um procedimento de construção de soluções iniciais semelhante ao proposto em Senarclens de Grancy e Reimann (2014a) e as metaheurísticas busca local iterada (Iterated local search - ILS) e busca local em vizinhança grande (Large neighborhood search - LNS) para melhoria dessas soluções. A abordagem híbrida, por sua vez, combina o algoritmo branchprice-and-cut (BPC) proposto em Munari e Morabito (2016) e as duas metaheurísticas supracitadas, resultando, desta forma, em duas abordagens híbridas, uma com a metaheurística ILS e a outra com a metaheurística LNS. Os resultados apresentados pelo autor indicam que a metaheurística ILS produz melhores resultados para o problema quando comparada à LNS, e quando comparada às abordagens propostas em Pureza, Morabito e Reimann (2012), fornecem também melhores soluções.

Nota-se que na grande maioria dos artigos no tema (PUREZA; MORABITO; REIMANN, 2012; DE GRANCY, 2015; DE GRANCY; REIMANN, 2016; MUNARI; MORABITO, 2016; ÁLVAREZ; MUNARI, 2017) pressupõe-se que os pontos de parada e o subconjunto de clientes em cada cluster tenham sido decididos previamente, estando o roteamento limitado aos veículos (rotas primárias). Na presente pesquisa, as decisões de segundo nível de roteamento (rotas secundárias, realizadas pelos entregadores) são incorporadas, caracterizando o problema como de Localização-roteamento em dois níveis com janelas de tempo e múltiplos entregadores (Two-echelon location-routing problem with time windows and multiple deliverymen - 2E-LRPTWMD).Especificamente, o 2ELRPTWMD visa obter a localização, o roteamento e a programação de pontos de parada dos veículos, o número de entregadores em cada veículo, assim como a designação, o roteamento e a programação do subconjunto de clientes servidos pelos entregadores a partir dos pontos de parada selecionados. Estas decisões devem ser tomadas de maneira a minimizar o custo total de atendimento de todas as demandas. Com vistas à representação e tratamento do problema, propõe-se um modelo de programação matemática para a situação em que o segundo nível de roteamento consiste de uma única rota por cluster, seguindo-se a resultados de experimentos com exemplos pequenos, com base em dados reais, e gerados a partir de instâncias clássicas do problema de roteamento de veículos com janelas de tempo.

Tanto quanto sabido, o modelo proposto é o primeiro esforço de representação do 
2E-LRPTWMD. Seu principal destaque é a consideração do tempo de serviço em cada cluster como uma variável explicitamente dependente das distâncias percorridas entre os clientes selecionados para compor o cluster.

\section{DESCRIÇÃO E FORMULAÇÃO DO PROBLEMA}

O 2E-LRPTWMD consiste em definir rotas primárias para uma frota de veículos homogênea de tamanho ilimitado que parte e retorna a um depósito central, assim como rotas secundárias para a tripulação dos veículos, composta por motoristas e ajudantes (Figura 1). As rotas primárias visitam um subconjunto de pontos de parada de veículos enquanto as rotas secundárias visitam clientes com demandas por produtos conhecidas, partindo e retornando aos pontos de parada visitados nas rotas primárias. No máximo uma única rota secundária emerge de cada ponto de parada e, como esperado, veículos e tripulação têm velocidades de deslocamento e capacidade de carregamento distintas.

Os locais de parada dos veículos consistem em um subconjunto de pontos cujas localizações são idênticas às dos clientes, ou seja, assume-se que os locais de parada sejam sempre em frente a algum cliente. Em cada rota primária, a carga total transportada não deve exceder a capacidade do veículo. O número de entregadores disponíveis é ilimitado, porém o tamanho da tripulação de cada veículo está restrito à capacidade da cabine. Em cada rota secundária, a demanda total do cluster servido não deve exceder a capacidade de transporte da tripulação, e a distância radial entre o ponto de parada e cada cliente do cluster não deve exceder um valor máximo. O tempo de rota está limitado à jornada de trabalho da tripulação, e janelas de tempo para início do serviço podem incorrer em um ou mais clientes.

Deseja-se obter os pontos de parada e clusters de clientes associados, o roteamento/programação das visitas, e os tamanhos de tripulação em cada veículo utilizado, de maneira que todos os clientes sejam atendidos, e que a solução resultante minimize o custo total da operação. O custo total é função do número de veículos utilizados, do número de pontos de parada visitados, do tempo total de rota e do número de entregadores utilizados. O número de pontos de parada visitados é um custo fictício que traduz a inconveniência de paradas dos veículos devido às dificuldades de circulação e estacionamento, estimulando o agrupamento de clientes, e consequente deslocamento dos entregadores em detrimento ao deslocamento dos veículos.

Seja $D=\{0\}$, o conjunto unitário correspondente ao depósito, $C=\{1,2, \ldots, n\}$ o conjunto de clientes a serem visitados, $S=\{n+1, n+2, \cdots\}$ o conjunto de pontos de paradas disponíveis e correspondentes a uma cópia do conjunto $C$ excluídas as cópias em que a parada de veículos não é possível, e $V=D \cup S \cup C$. Seja também $A_{1}$ o conjunto de arcos direcionados entre cada par $i_{z} j \in D \cup S$ e $A_{2}$ o conjunto de arcos direcionados entre cada par $i_{y} j \in S \cup C$. $O$ problema pode ser representado pelos grafos conexos $G_{1}=\left\{D \cup S, A_{1}\right\}$ e $G_{2}=\left\{S \cup C, A_{2}\right\}$. Ou seja, o conjunto $A_{1}$ corresponde aos arcos que podem ser percorridos somente por veículos (rotas primárias, envolvendo o depósito e pontos de parada) e o conjunto $A_{2}$ corresponde aos arcos que podem ser percorridos somente por entregadores (rotas secundárias, envolvendo pontos de parada e clientes). A cada $\operatorname{arco}(i, j) \in A_{1}$ há uma distância associada e correspondente ao comprimento do caminho mais curto entre $i$ e $j$, considerando a malha viária em questão. Dado que o deslocamento dos entregadores não sofre as restrições da malha viária enfrentadas pelos veículos, o comprimento do $\operatorname{arco}(i, j) \in A_{2}$ corresponde à distância euclidiana entre $i$ e $j$.

Para cada nó $i \in C$ há uma demanda associada pelo produto e tempos de serviço, os quais são função do número de entregadores empregados (se o número de entregadores é $l$, 
diz-se que o veículo viaja em modo $l$ ). Por simplicidade, considera-se que tempos de serviço com mais de um entregador são obtidos dividindo-se o tempo com um entregador pelo número de entregadores. Adicionalmente, associa-se uma janela de tempo a cada nó $i \mathrm{U} V$; se $i=D$, os limites das janelas são iguais aos instantes de abertura e fechamento do depósito, enquanto para $i \in C$, os limites indicam o intervalo de tempo em que o serviço em $i$ deve ser necessariamente iniciado.

Seja a seguinte notação:

$\underline{\text { Variáveis de decisão }}$

$x_{i j l}=1$ se um veiculo percorre o arco $i-j$ em uma rota primária no modo $l ; 0$, caso contráric $x s_{i j k l}=1$ se a tripulação percorre o arco $i-j$ na rota secundária que se inicia no ponto de parada $k$ no mode $l ; 0$, caso contráric

$H_{i k}=1$ se c cliente $i$ é designado ao cluster servido a partir do ponto de parada $k$; 0 , caso contrário

$T_{i l}$ : instante em que o veículo chega ao nó $i$ no modo $l$

$T F_{k l}$ instante em que a tripulação do veículo que visita o ponto de parada $k$ em modo $l$ retorna a $k$ após a finalização da rota secundária

$T S x_{k l}$ : tempo de serviço no cluster servido a partir do ponto de parada $k$ no modo $l$ $Y_{i l}$ : carga no veículo após servir o ponto de parada $i$ em uma rota primária no modo $l$ $Q A_{i^{\sharp}}$ demanda do cluster servido a partir do ponto de parada $i$

\section{Parâmetros}

$C_{1}$ : custo fixo de um veículo da frota

$C_{2}$ : custo de uma unidade de tempo de deslocamento de um veículo da frota

$C_{3}$ : custo de uma parada de veículo

$C_{4}:$ custo de um entregador

$c a_{i j}$ : distância entre $i$ e $j,(i, j) \in A_{1}$, percorrida por veículos da frota

$c b_{i j}$, distância entre $i$ e $j,(i, j) \in A_{2}$, percorrida por equipes de entregadores

$v_{1}$ : velocidade de deslocamento de veículos da frota

$v_{2}$ : velocidade de deslocamento de equipes de entregadores

$t v 1_{i j}$ : tempo de viagem entre $i \mathrm{e} j,(i, j) \in A_{1}$, de veículos da frota

$t v 2_{i j}$ : tempo de viagem entre $i$ e $j,(i, j) \in A_{2}$, de equipes de entregadores

$Q_{i}$ : demanda de $i \in C$

$Q D_{l^{\text {s }}}$ capacidade de carga de uma equipe de entregadores no modo $l$

QT: capacidade de carga de um veículo da frota

$T S_{i l}$ tempo de serviço de $i \in C$ no modo $l$

$\left[a_{i}, b_{i}\right]$ : janela de tempo em $i \in V$

maxdist: distância radial máxima entre um ponto de parada e um cliente do cluster associado

$M_{i k l}^{1}=b_{i}+T S_{i l}+t v 1_{i j}-a_{j}$ se $b_{i}+T S_{i l}+t v 1_{i j}-a_{j} \geq 0 ; 0$ caso contrário

$M_{i k l}^{2}=b_{i}+T S_{i l}+t v 2_{i j}-a_{j}$ se $b_{i}+T S_{i l}+t v 2_{i j}-a_{j} \geq 0 ; 0$ caso contrário

O problema é formulado como um modelo de programação inteira mista, conforme descrito a seguir. Considera-se também que algumas variáveis são previamente fixadas. Por exemplo, fixa-se $x_{i j l}=0$ se $i=j$ ou $x s_{i j k l}=0$ se $k \notin S$, dentre outras condições.

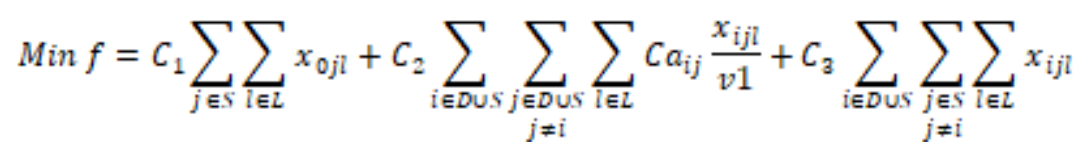




$$
\begin{aligned}
& +C_{4} \sum_{j \in 5}\left(1 x_{0 j 1}+2 x_{0 j 2}+3 x_{0 j 1}\right) \\
& \sum_{i \in D u S} \sum_{i \in L} x_{i j l} \leq 1 \quad \forall j \in S \\
& \sum_{j \in D \cup S}^{i \neq j} \sum_{i \in L} x_{i j 1} \leq 1 \quad \forall i \in S \\
& \sum_{j \in D U S}^{j \neq i} x_{j i l}=\sum_{j \in D U S} x_{i j l} \quad \forall i \in S, \forall l \in L \\
& x s_{k j k l}^{j \neq i} \leq \sum_{\substack{i \in D U S \\
i \neq k}}^{j \neq i} x_{i k l} \quad \forall k \in S, \forall j \in C, \forall l \in L \\
& \sum_{j \in C} x s_{k j k l} \geq \sum_{\substack{i \in D \\
i \neq k}} x_{i k \mathbb{R}} \quad \forall k \in S, \forall l \in L \\
& \sum_{i \in S \cup C} \sum_{k \in S} \sum_{i \in L} x s_{i j k l}^{i \neq k}=1 \quad \forall j \in C \\
& \sum_{j \in S U C}^{i \neq j} \sum_{k \in S} \sum_{i \in L} x s_{i j k l}=1 \quad \forall i \in C \\
& \sum_{j \in C}^{j \neq i} x s_{k j k l}=\sum_{j \in C} x s_{j k k l} \quad \forall k \in S, \forall l \in L \\
& \sum_{\substack{j \in C \\
\text { orj } j=k}} x s_{j i k l}=\sum_{\substack{j \in C \\
\text { orj }=k}} x s_{i j k l} \quad \forall i \in C_{v} \forall k \in S, \forall l \in L \\
& \sum_{j \in V}^{\circ \operatorname{orj} j=k} \sum_{i \in L}^{k} x s_{k j k l} \leq 1 \quad \forall i \in S \\
& \sum_{j \in S} H_{i j}=1 \quad \forall i \in C \\
& n \sum_{i \in D \cup S} \sum_{i \in L} x_{i j p} \geq \sum_{i \in C} H_{i j} \quad \forall j \in S \\
& \sum_{\substack{i \in D u S \\
i \neq j}}^{i \neq j} \sum_{i \in L} x_{i j i} \leq \sum_{i \in C} H_{i j} \quad \forall j \in S \\
& \sum_{j \in \in \cup \cup[k,} \sum_{i \in L} x S_{j i k l}=H_{i k} \quad \forall i \in C_{s} \forall k \in S \\
& \sum_{j \in C \cup[k]} \sum_{i \in L} x s_{i j k l}=H_{i k} \quad \forall i \in C, \forall k \in S \\
& Q A_{k}=\sum_{i \in C} Q_{i \mathrm{i}} H_{\mathrm{ik}} \quad \forall k \in S \\
& Q A_{k} \leq \sum_{i \in C} \sum_{i \in L} Q D_{i} x_{i k n} \quad \forall k \in S \\
& C b_{i k} H_{i k} \leq \text { maxdist } \quad \forall i \in C, \forall k \in S \\
& T_{j p} \geq T_{i l}+T S x_{i \mathbb{l}}+t v 1_{i j} x_{i j p}-M_{i j p}^{1}\left(1-x_{i j l}\right) \quad \forall i \in S, \forall j \in D \cup S_{v} j \neq i, \forall l \in L \\
& T_{j l} \geq T_{k l}+t v 2_{k j l} x s_{k j k l}-M_{k j p}^{2}\left(1-x s_{k j k l}\right) \quad \forall j \in C, \forall k \in S, \forall l \in L \\
& T_{j 1} \geq T_{i 1}+\left(T S_{i j}+t v 2_{i j} x s_{i j k l}\right)-M_{k j p}^{2}\left(1-x s_{i j k l}\right) \quad \forall i \in C, \forall j \in C_{v} j \neq i, \\
& \forall k \in S, \forall l \in L \\
& T F_{k l} \geq T_{i l}+\left(T S_{i \mathbb{1}}+t v 2_{i k}\right) x s_{i k k l}-M_{i k l}^{2}\left(1-x s_{i k k l}\right) \quad \forall i \in C, \forall k \in S, \forall l \in L \\
& T S_{k l}=T F_{k l}-T_{k l} \quad \forall k \in S, \forall l \in L
\end{aligned}
$$




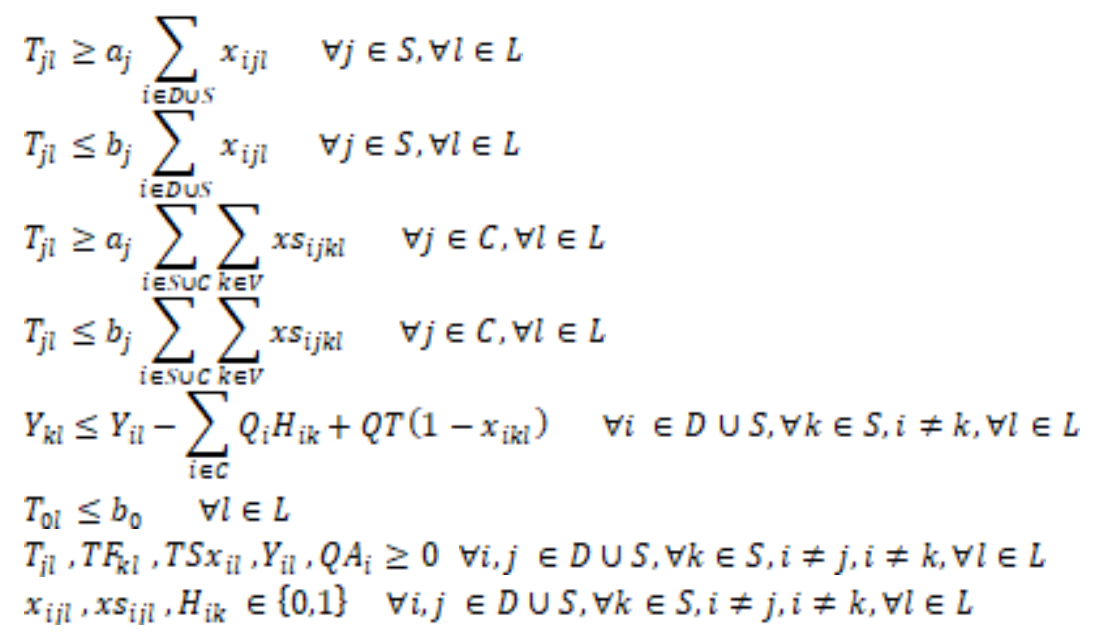

A função objetivo a ser minimizada (1) consiste na soma dos custos que incidem sobre os veículos utilizados, tempo total de rota, número de paradas dos veículos e número de entregadores empregados. As restrições (2) e (3) garantem que cada ponto de parada deve ser visitado no máximo uma vez. As restrições (4) impõem a conservação de fluxo, prescrevendo que o mesmo veículo que chega a um ponto de parada em um modo $l$, parte desse ponto no mesmo modo $l$. As restrições (5) impedem rotas secundárias a partir de um ponto de parada $k$ se este não for visitado em uma rota primária. As restrições (6) prescrevem que se um ponto de parada $k$ for visitado em uma rota primária pelo menos uma rota secundária se origina dele.

As restrições (7) e (8) impõem que cada cliente seja visitado exatamente uma vez em uma rota secundária. As restrições (9) impõem a conservação de fluxo nas rotas secundárias, assegurando que o mesmo número de rotas secundárias que se originam em um ponto de parada $k$ em um modo $l$ deve retornar a $k$ no mesmo modo $l$. As restrições (10) impõem que uma tripulação que chega a um cliente $i \mathrm{em}$ um modo $l$ deve partir de $j$ no mesmo modo $l$. As restrições (11) prescrevem que no máximo uma rota secundária se origine de cada ponto de parada. As restrições (12) impõem que cada cliente seja designado a um único ponto de parada/cluster. As restrições (13) forçam que se pelo menos um cliente for designado a um ponto de parada $j$ então $j$ é visitado em uma rota primária.

As restrições (14) impõem que se nenhum cliente for designado a um ponto de parada $k, k$ não é visitado em uma rota primária. As restrições (15) e (16) prescrevem que se um cliente $i$ for designado a um ponto de parada $k$, $i$ é visitado em uma rota secundária que se origina em $k$. As restrições (17) calculam a demanda de cada cluster de clientes servidos a partir do ponto de parada $k$ como a soma das demandas individuais desses clientes.

As restrições (18) limitam a demanda de cada cluster à capacidade de carga dos entregadores em uma única rota secundária. As restrições (19) especificam a distância máxima radial entre cada cliente de um cluster e o ponto de parada associado.

As restrições (20) calculam os instantes de chegada aos nós das rotas primárias, considerando que o tempo de serviço no cluster de clientes servidos a partir do ponto de parada $k$ no modo $l\left(T S x_{k l}\right)$ é uma variável que depende de $l$ e do tempo dispendido na rota secundária que se origina em $k$. A programação dos $l$ entregadores em cada rota secundária é feita da seguinte forma: as restrições (21) computam o instante de chegada ao primeiro cliente visitado, as restrições (22) computam os instantes de chegada aos demais clientes, as restrições (23) calculam o instante de finalização da rota secundária $\left(T F_{k l}\right)$, ou seja, o instante de retorno ao ponto de parada associado $k$, e as restrições (24) computam $T S x_{k l}$ como a diferença entre $T F_{k l}$ e o instante de chegada a $k$ na rota primária $T_{k l}$. 
As restrições (25) e (26) ativam as janelas de tempo dos pontos de parada nas rotas primárias, enquanto as restrições (27) e (28) ativam as janelas de tempo dos clientes nas rotas secundárias. As restrições (29) computam a carga do veículo após a visita a cada ponto de parada nas rotas primárias e as restrições (30) fornecem a duração máxima de cada rota primária. Por fim, as restrições (31) e (32) impõem os domínios das variáveis de decisão.

\section{EXPERIMENTOS COMPUTACIONAIS}

Esta seção descreve os experimentos computacionais realizados com o modelo (1)(32), inicialmente com exemplos de pequeno porte e quatro conjuntos de exemplos gerados a partir de dados reais de uma empresa distribuidora de bebidas. O modelo foi implementado na linguagem de modelagem GAMS e resolvido com o solver CPLEX 12.5 por um tempo de processamento máximo de 18.000 segundos. Todos os experimentos foram realizados em um computador Dell Optiplex 9010, processador principal Intel Core i7 com 3,4 GHz, 16 GB de memória RAM e sistema operacional Windows 7 Professional de 64 bits.

Com base no estudo de caso de Souza Neto e Pureza (2016), foram adotados seguintes parâmetros constantes no modelo: $C_{1}=1000, C_{2}=1,1, C_{3}=500$ e $C_{4}=100$. Ressalta-se que enquanto $C_{1}, C_{2}$ e $C_{4}$ representam de forma razoável as grandezas dos custos na prática das empresas distribuidoras de bebidas, $C_{3}$ foi concebido de forma a valorizar 0 deslocamento dos entregadores sempre que possível.

\subsection{InSTÂnCIAS de PEQUeno PORTE (toys)}

Este conjunto de experimentos teve como objetivo tanto a validação do modelo como a análise mais detalhada de possíveis padrões nas soluções e na convergência do método de solução quando alguns parâmetros do problema são variados. Para tal, um conjunto de sete instâncias toy de 12 clientes foram produzidas com base em abordagens de geração da literatura, e modificadas em concordância com as características do 2E-LRPTWMD.

Especificamente, as coordenadas do depósito e dos clientes foram geradas entre 0 e 100 unidades de distância segundo a abordagem proposta em Uchoa et al. (2017) para distribuição espacial agrupada, as demandas seguem uma distribuição uniforme entre os valores 10 e 40 unidades, as janelas de tempo (quando existentes) foram geradas com base na abordagem proposta por Solomon (1987), e os tempos de serviço para cada cliente foram calculados de acordo com Pureza, Morabito e Reimann (2012).

Todos os toys compartilham a mesma distribuição geográfica de clientes (Figura 2), demandas, capacidade dos veículos, e janela de tempo do depósito $([\mathrm{a} 0, \mathrm{~b} 0]=[0,280])$. As distâncias entre nós são simétricas e foram calculadas segundo a métrica euclidiana para o caso de percursos realizados por entregadores. Para percursos realizados por veículos, as distâncias são também simétricas, porém a distância é um múltiplo igual a 1 ou 3 da distância euclidiana, a fim de simular uma possível situação em uma malha viária urbana. Os dados de entrada restantes são específicos para cada toy e descritos a seguir. 


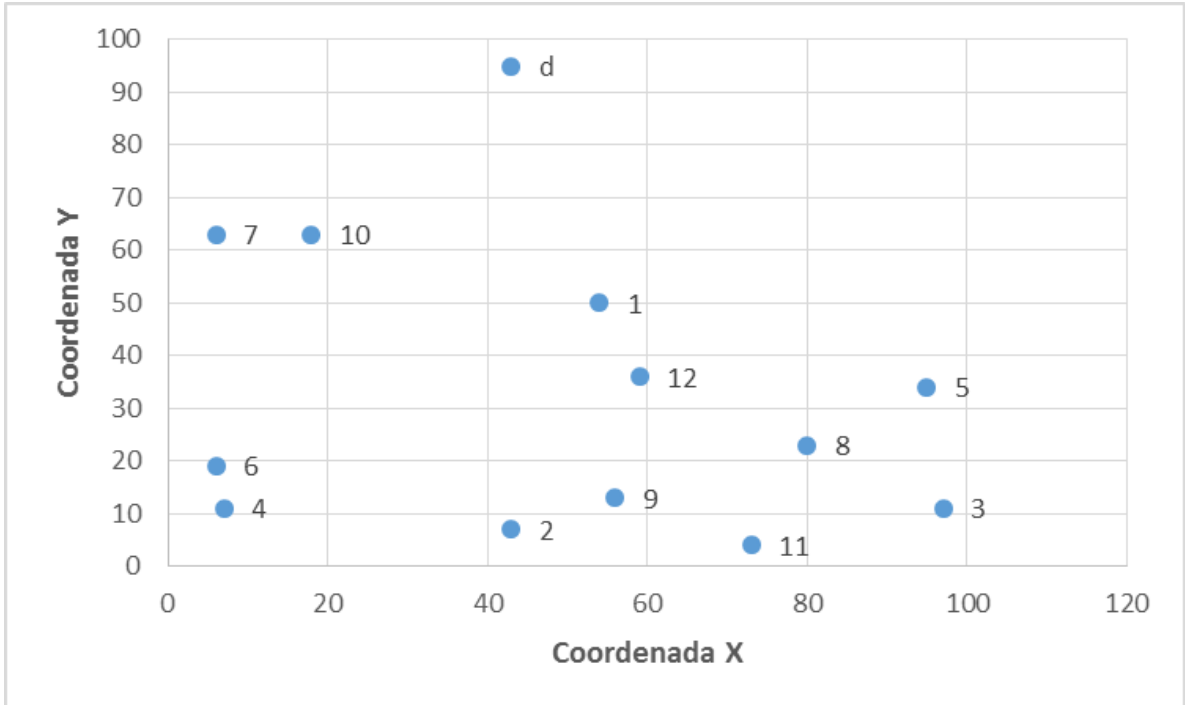

Figura 2 - Distribuição geográfica dos clientes nos toys.

\subsubsection{Resultados}

Toy 1

Os dados específicos do exemplo são apresentados no Quadro 1. Nota-se que corresponde a uma instância pouco restrita, pois não há janelas de tempo em clientes e todos os pontos de parada estão disponíveis. A distância radial para agrupamento (150 unidades de distância), permite, a princípio, a formação de clusters com vários clientes.

\section{Quadro 1 - Dados específicos de toy 1.}

\begin{tabular}{|c|c|c|c|}
\hline $\boldsymbol{i}$ & $\boldsymbol{a}_{\boldsymbol{i}}$ & $\boldsymbol{b}_{\boldsymbol{i}}$ & maxdist \\
\hline $1-12$ & 0 & 280 & 150 \\
\hline
\end{tabular}

A Figura 3 ilustra as rotas obtidas. As diferentes cores das linhas distinguem as rotas, com as linhas sólidas indicando o percurso dos veículos (rotas primárias) e as linhas tracejadas o percurso dos entregadores (rotas secundárias). A solução utiliza 3 veículos, cada qual atendendo 1 cluster. As rotas em laranja, verde e cinza utilizam respectivamente, 3, $2 \mathrm{e}$ 2 entregadores (por simplicidade, neste e nos demais toys não serão reportadas as distâncias de cada rota). Observa-se que o solver não provou a otimalidade da solução (gap de 22,6\%), o que revela as dificuldades de convergência do método de solução quando a instância é pouco restrita, mesmo quando seu porte é pequeno. 


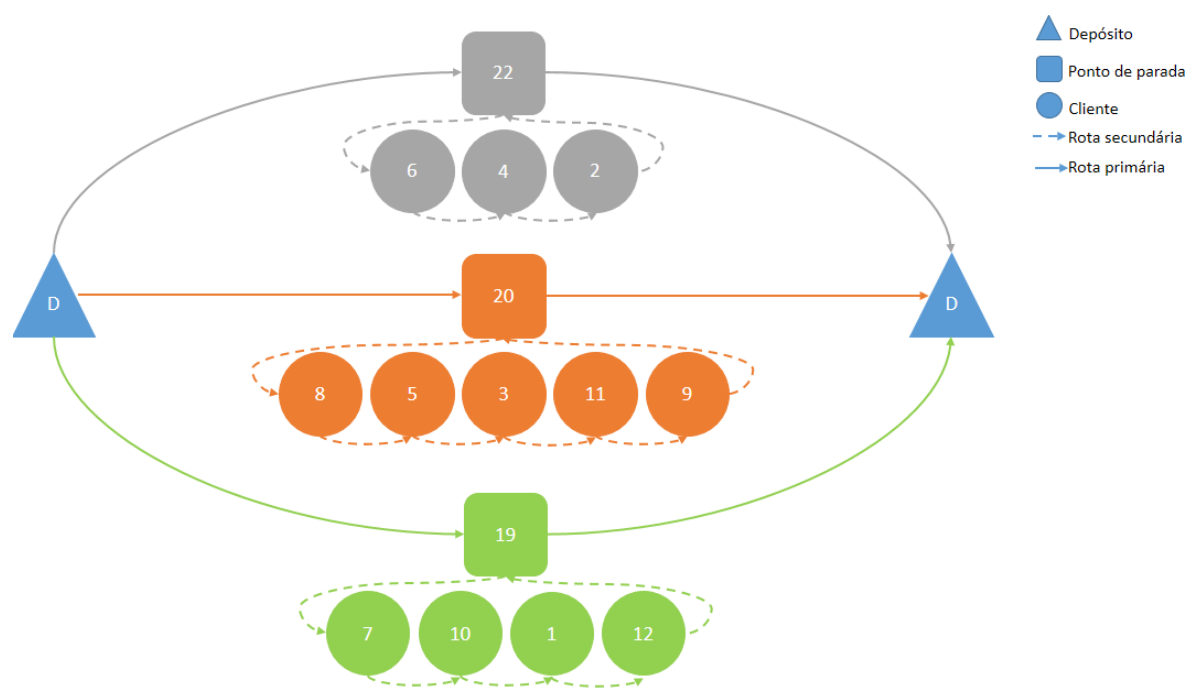

Figura 3 - Rotas de toy 1.

\section{Toy 2}

Este exemplo tem os mesmos dados de toy 1, com exceção da imposição da indisponibilidade de utilização dos pontos de parada em frente aos clientes 2,4 e 8 (coluna $\mathrm{U}$ do Quadro 2).

Quadro 2 - Dados específicos de toy 2.

\begin{tabular}{|c|c|c||c|c|}
\hline $\boldsymbol{i}$ & $\boldsymbol{a}_{\tilde{\boldsymbol{i}}}$ & $\boldsymbol{b}_{\tilde{\boldsymbol{i}}}$ & maxdist & $\mathbf{U}$ \\
\hline $1-12$ & 0 & 280 & 150 & $2,4,8$ \\
\hline
\end{tabular}

A Figura 4 ilustra as rotas obtidas. São também utilizados 3 veículos, cada um atendendo 1 cluster de clientes. As rotas em laranja, verde e cinza têm, respectivamente, 2, 2 e 3 entregadores. O gap é igual a $22,42 \%$, o que indica que a indisponibilidade de alguns pontos de parada não trouxe ganhos substanciais na convergência do método.

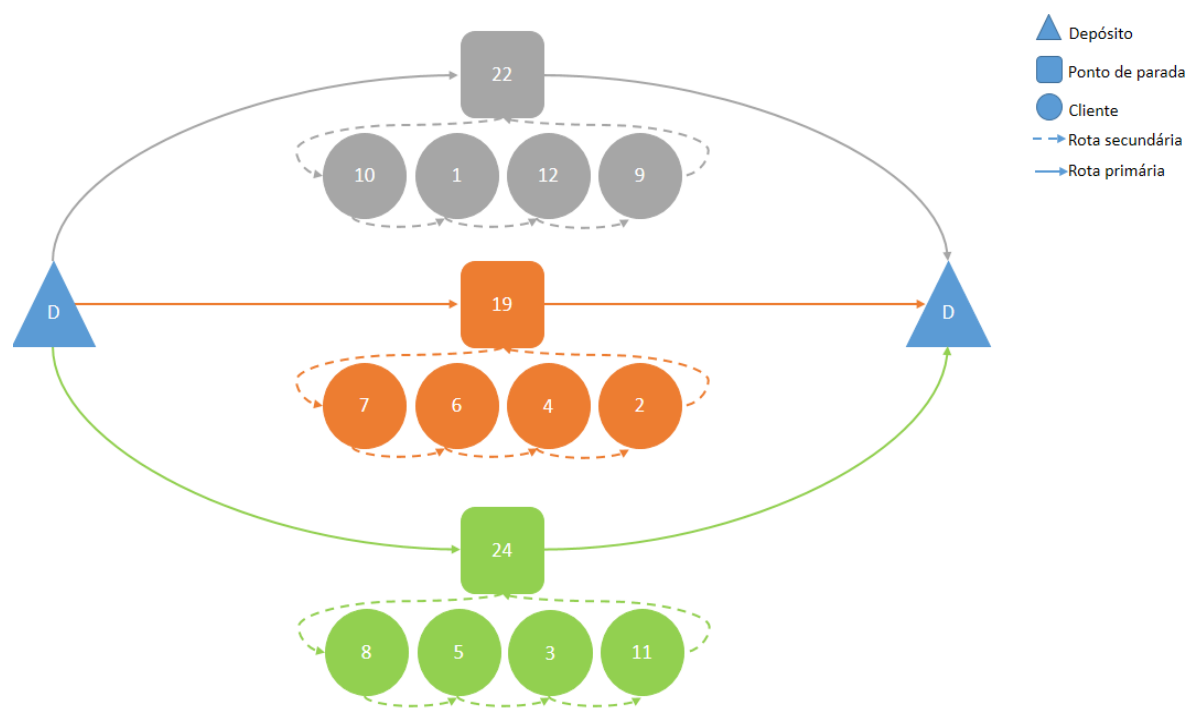

Figura 4 - Rotas de toy 2. 


\section{Toy 3}

Este exemplo tem os mesmos dados de toy 2 (inclusive o conjunto de pontos de parada indisponíveis), e a inclusão de janelas de tempo nos clientes 5, 6, 7 e 8 e nos pontos de parada em frente a estes clientes (Quadro 3). Neste e demais exemplos, o instante de abertura da janela de um dado ponto de parada é igual ao do cliente e o instante de encerramento é igual ao do depósito.

Quadro 3 - Dados específicos de toy 3.

\begin{tabular}{|c|c|c||c|c|}
\hline $\boldsymbol{i}$ & $\boldsymbol{a}_{\boldsymbol{i}}$ & $\boldsymbol{b}_{\boldsymbol{i}}$ & maxdist & $\mathbf{U}$ \\
\hline $1-4,9-12$ & 0 & 280 & 150 & $2,4,8$ \\
\hline $5-8$ & Entre 35 e 90 & Entre 40 e 95 & & \\
\hline
\end{tabular}

A Figura 5 ilustra a solução obtida, a qual também utiliza 3 veículos, atendendo 1 cluster cada. Nota-se que o gap é igual a $17,19 \%$, o que sugere que a inclusão de janelas de tempo em alguns clientes melhora ligeiramente a convergência do método.

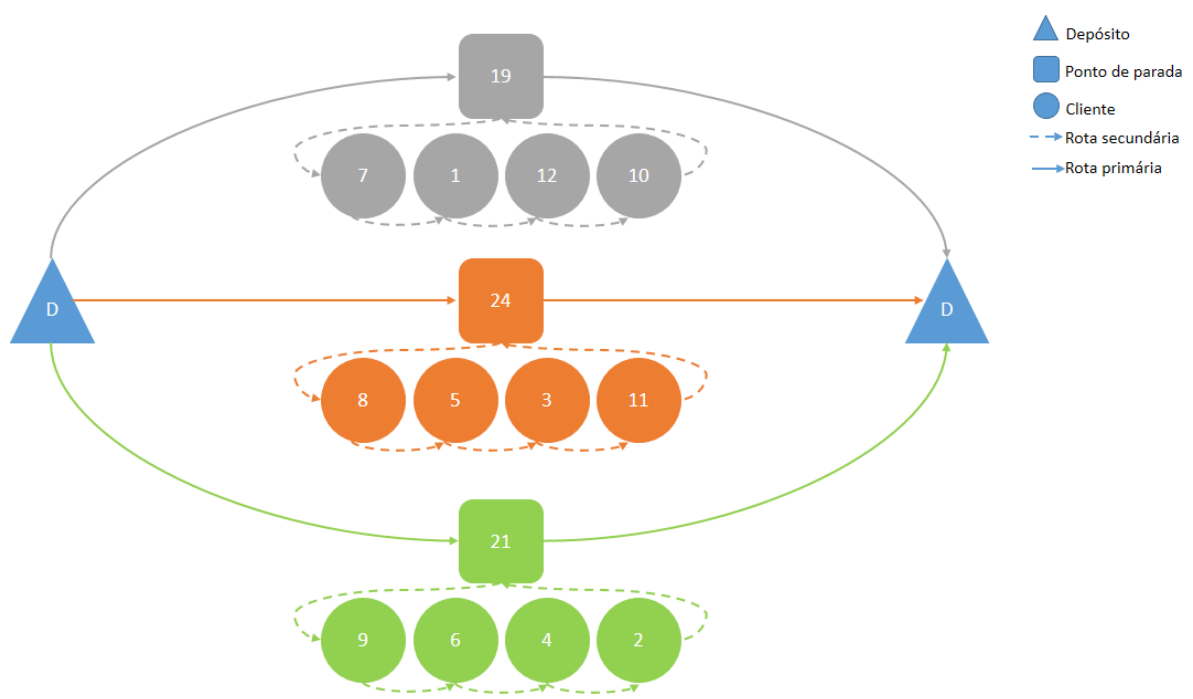

Figura 5 - Rotas de toy 3.

\section{Toy 4}

Neste exemplo, os mesmos dados de toy 3 foram utilizados, exceto que todos os clientes possuem janelas de tempo. Os dados adicionais em relação aos compartilhados por todas as instâncias são apresentados no Quadro 4 a seguir.

\section{Quadro 4 - Dados específicos de toy 4.}

\begin{tabular}{|c|c|c||c|c|}
\hline $\boldsymbol{i}$ & $\boldsymbol{a}_{\boldsymbol{i}}$ & $\boldsymbol{b}_{\boldsymbol{i}}$ & maxdist & $\mathbf{U}$ \\
\hline $1-12$ & Entre 10 e 165 & Entre 15 e 170 & 150 & $2,4,8$ \\
\hline
\end{tabular}

A Figura 6 ilustra a solução obtida, a qual utiliza 3 veículos e atende 5 clusters com 6 entregadores. Esta solução é comprovadamente ótima e foi obtida em 7 segundos de processamento, revelando que a imposição de janelas de tempo para todos os clientes 
aumentou muito a velocidade de convergência do método. Nota-se que nas rotas verde e cinza há clusters unitários, contemplando respetivamente, os clientes 7 e 10. Observa-se também que para servir o cluster formado pelos clientes 3, 5, 8 e 11, o veículo estaciona no ponto de parada em frente ao cliente 5 , a partir do qual, os entregadores atendem o cliente 8 para só então atenderem o cliente 5. Isso pode ser explicado pelo fato da janela de cliente 8 $([35,40])$ preceder a janela do cliente $5([75,87])$.

4.

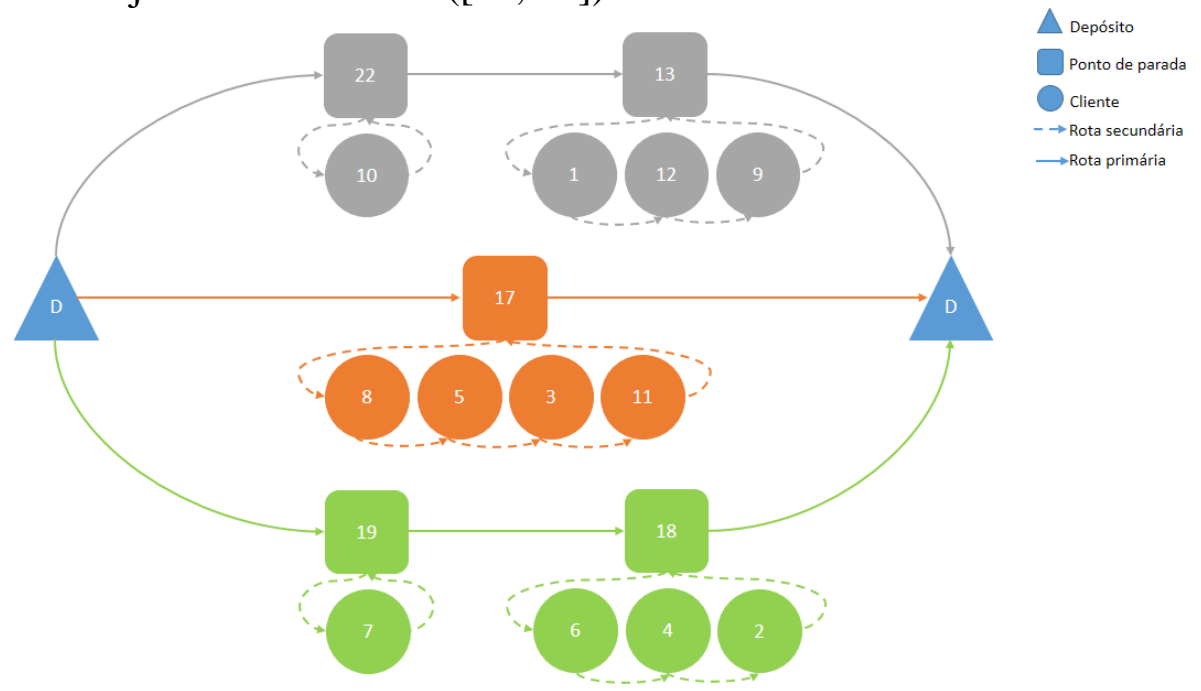

Figura 6 - Rotas de toy 4.

\section{Toy 5}

Este exemplo possui as mesmas janelas de tempo e indisponibilidade de pontos de parada de toy 3, porém é feita uma redução de $60 \%$ na máxima distância radial entre um cliente de um cluster e o ponto de parada do veículo que o serve (Quadro 5).

Quadro 5 - Dados específicos de toy 5.

\begin{tabular}{|c|c|c|c|c|}
\hline $\boldsymbol{i}$ & $\boldsymbol{a}_{\boldsymbol{i}}$ & $\boldsymbol{b}_{\boldsymbol{i}}$ & maxdist & $\mathbf{U}$ \\
\hline $1-4,9-12$ & 0 & 280 & 60 & $2,4,8$ \\
\hline $5-8$ & Entre 35 e 90 & Entre 40 e 95 & & \\
\hline
\end{tabular}

A solução incumbente é idêntica à solução de toy 3, entretanto, seu gap é menor $(16,57 \%)$. Ressalta-se que esta máxima distância adotada não restringe, na prática, qualquer agrupamento.

\section{Toy 6}

Neste exemplo, são adotados os dados de toy 3 e realizada uma redução ainda maior (80\%) da máxima distância radial entre um cliente e o ponto de parada do cluster (Quadro 6). A Figura 7 ilustra as rotas obtidas.

Quadro 6 - Dados específicos de toy 6.

\begin{tabular}{|c|c|c||c|c|}
\hline $\boldsymbol{i}$ & $\boldsymbol{a}_{\boldsymbol{i}}$ & $\boldsymbol{b}_{\boldsymbol{i}}$ & maxdist & $\mathbf{U}$ \\
\hline $1-4,9-12$ & 0 & 280 & 30 & $2,4,8$ \\
\hline $5-8$ & Entre 35 e 90 & Entre 40 e 95 & & \\
\hline
\end{tabular}




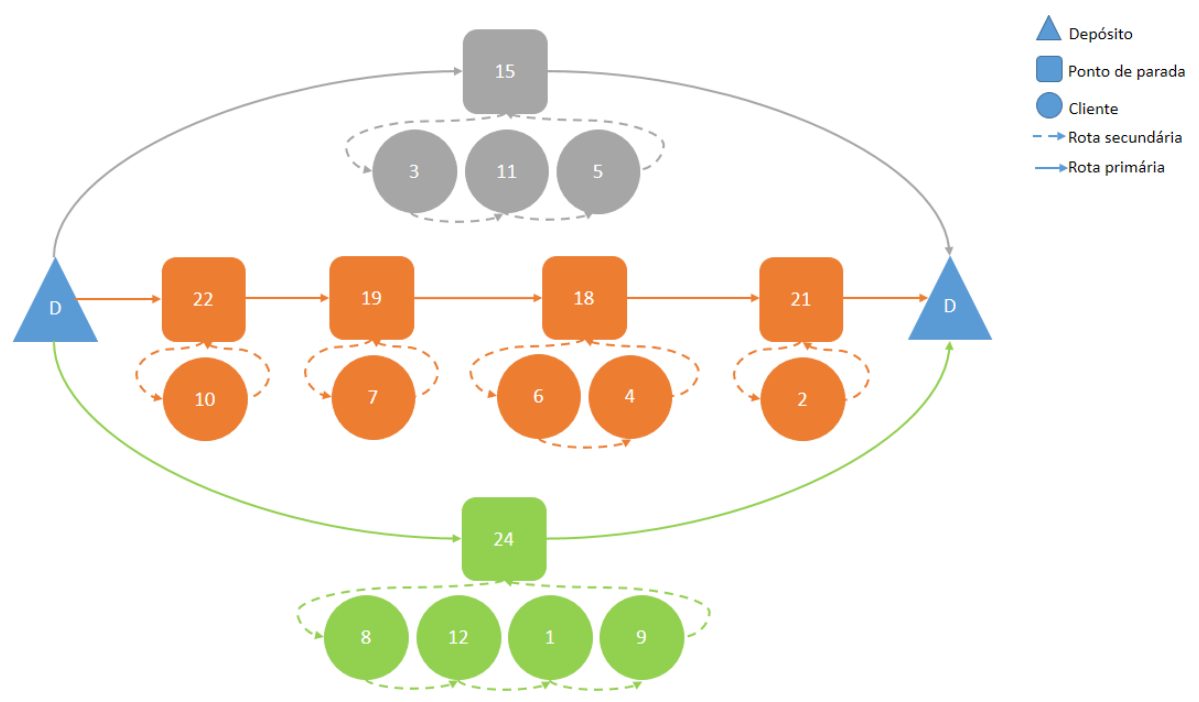

Figura 7 - Rotas de toy 6.

A solução utiliza 3 veículos, 9 entregadores, e atende 6 clusters. Esta solução é ótima e foi obtida após 197 segundos de execução, o que indica que a diminuição da máxima distância radial para pertinência de clientes em clusters pode facilitar a convergência do método de solução, pelo menos para instâncias de pequeno porte. Note que o número de clusters é maior que o obtido com o toy anterior, uma vez que um número menor de clientes pode ser designado a um mesmo cluster.

\section{Toy 7}

Este exemplo possui os mesmos dados que toys 3, 5 e 6, com exceção da máxima distância radial para inclusão de clientes em clusters, reduzida para 21 unidades (Quadro 7). A Figura 8 ilustra as rotas obtidas. A solução foi obtida em 18 segundos de processamento, é ótima, faz uso de 3 veículos, 6 entregadores e serve 8 clusters.

\section{Quadro 7 - Dados específicos de toy 7.}

\begin{tabular}{|c|c|c|c|c|}
\hline $\boldsymbol{i}$ & $\boldsymbol{a}_{\boldsymbol{i}}$ & $\boldsymbol{b}_{\boldsymbol{i}}$ & maxdist & $\mathbf{U}$ \\
\hline $1-4,9-12$ & 0 & 280 & 21 & $2,4,8$ \\
\hline $5-8$ & Entre 35 e 90 & Entre 40 e 95 & & \\
\hline
\end{tabular}




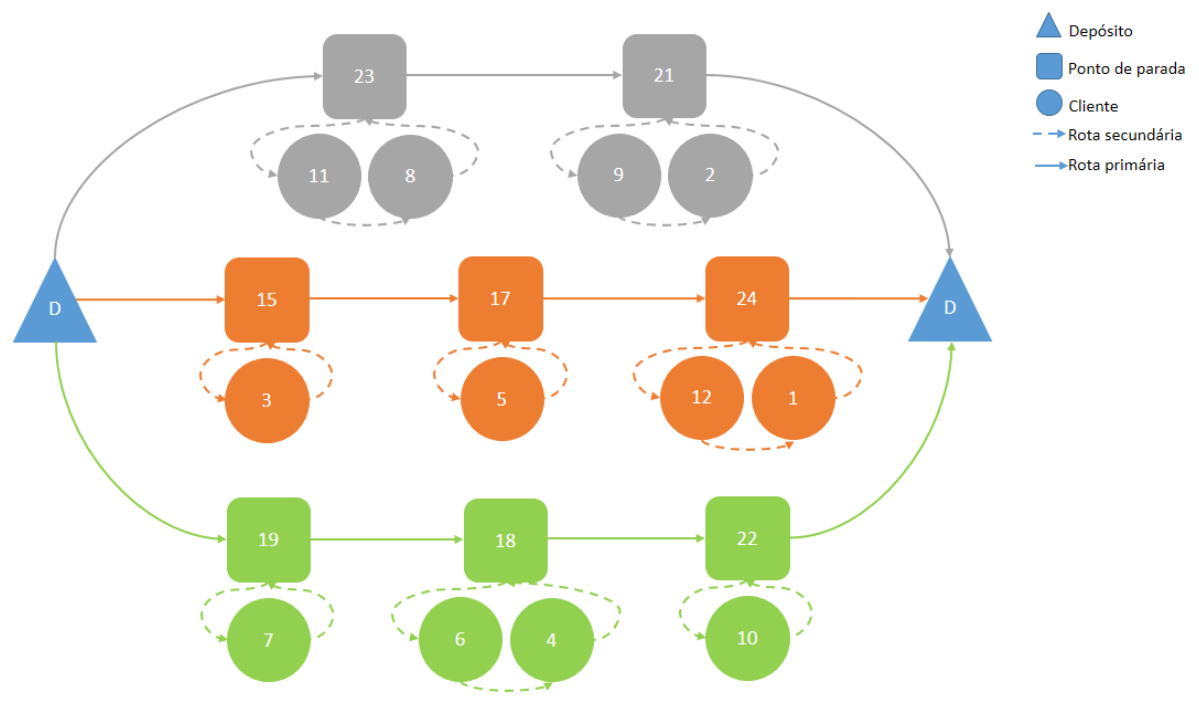

Figura 8 - Rotas de toy 7.

Os resultados dos toys 3, 5, 6 e 7 permitem concluir que à medida que a máxima distância radial para agrupamento é reduzida diminui-se também o número de clientes por cluster, o que leva à anulação de muitas variáveis de fluxo em rotas secundárias. Como decorrência, os exemplos tendem a ser resolvidos otimamente mais rapidamente.

\subsection{INSTÂNCIAS DE MAIOR PORTE}

Nestes experimentos, são contemplados conjuntos de exemplos entre 15 a 50 clientes (empresas varejistas abastecidas por uma distribuidora de bebidas) de uma cidade de médio porte do interior do Estado de São Paulo. Os dados coletados são os endereços, demandas e tempos de serviço de clientes, enquanto as distâncias para veículos e entregadores foram obtidas com o serviço de pesquisa e visualização de mapas Google Maps. As velocidades utilizadas são a velocidade média de um veículo trafegando em cidades com congestão veicular $\left(v_{1}=24 \mathrm{~km} / \mathrm{h}\right)$ e a velocidade média de um indivíduo caminhando $\left(v_{2}=5 \mathrm{~km} / \mathrm{h}\right)$. O instante de abertura do depósito é 0 e seu encerramento é 28.800 segundos, equivalentes a 8 horas de jornada diária de trabalho. As janelas de tempo para os clientes e pontos de parada são triviais e amplas (intervalos definidos por $a_{i}=a_{1}+t v 1_{1 i}(i \in S \cup C), b_{i}=b_{1}-t v 1_{i 1}-T S_{i 1}(i \in C)$ e $b_{i}=b_{1}(i \in S)$ ), de forma a garantir a existência de soluções factíveis em cada exemplo.

Tabela 1: Resultados computacionais com instâncias de maior porte.

\begin{tabular}{|c|c|c|c|c|c|c|c|c|}
\hline Conjunto & $n$ & $\begin{array}{c}\mathbf{N}^{\circ} \text { de } \\
\text { exemplos }\end{array}$ & Gap $(\%)$ & $\begin{array}{c}\text { Tempo } \\
(\mathrm{s})\end{array}$ & $\begin{array}{c}\text { Distância } \\
(\mathrm{m})\end{array}$ & $\begin{array}{c}\mathbf{N}^{\circ} \mathrm{de} \\
\text { clusters }\end{array}$ & $\begin{array}{c}\mathrm{N}^{\circ} \mathrm{de} \\
\text { veículos }\end{array}$ & $\begin{array}{c}\mathrm{N}^{\circ} \text { de } \\
\text { entregadores }\end{array}$ \\
\hline 1 & 15 & 6 & 0 & 35,1 & 11363,7 & 7,2 & 1,2 & 2,6 \\
\hline 2 & 20 & 6 & 0 & 901 & 17311,2 & 9,7 & 2 & 3,7 \\
\hline 3 & 25 & 3 & 2,9 & 14276,6 & 16240,0 & 9,0 & 2,0 & 4,7 \\
\hline 4 & 50 & 1 & 4,9 & 18000 & 34233,1 & 21 & 4 & 10 \\
\hline 5 & 12 & 3 & 32,2 & 18000 & 43,1 & 2,7 & 3,0 & 5,7 \\
\hline 6 & 25 & 3 & 55,6 & 18000 & 84,2 & 7,0 & 7,0 & 12,3 \\
\hline $7^{\diamond}$ & 50 & 3 & 65,1 & 18000 & 517,7 & 34,0 & 34,0 & 54,0 \\
\hline
\end{tabular}

${ }^{\diamond}$ Média de resultados de dois dos três exemplos 
As quatro primeiras linhas da Tabela 1 apresentam os resultados médios de cada conjunto, os quais sugerem que métodos exatos de softwares comerciais são capazes de obter boas soluções no tempo de processamento disponibilizado. De fato, o solver CPLEX conseguiu resolver otimamente todos os exemplos até 20 clientes e um exemplo de 25 clientes, enquanto gaps individuais inferiores a $9 \%$ foram obtidos para os demais exemplos.

A fim de verificar se este comportamento se mantinha para outros tipos de instâncias, experimentos adicionais foram realizados com três conjuntos de exemplos $(12,15$ e 50 clientes), gerados a partir das instâncias RC101, RC102 e RC103 propostas em Solomon (1987) para o problema de roteamento de veículos com janelas de tempo. Cada conjunto contempla três exemplos, dos quais foram mantidas as coordenadas originais do depósito e dos clientes, assim como as demandas dos clientes. As matrizes de distâncias (assimétricas) percorridas por veículos e por entregadores foram geradas uniformemente nos intervalos $[0,100]$ e $[0,50]$, respectivamente. As janelas de tempo dos clientes e demais parâmetros foram calculados de forma similar aos dos conjuntos 1 a 4 .

Diferentemente dos resultados anteriores, as soluções obtidas para 8 dos 9 exemplos dos conjuntos 5 a 7 apresentam gaps substancialmente altos. Em 6 casos os gaps são superiores a $20 \%$, mesmo após 5 horas de processamento, enquanto que para um exemplo de 50 clientes, o solver CPLEX não foi capaz de obter qualquer solução factível dentro do tempo limite.

\section{CONCLUSÕES}

Este trabalho endereçou as operações de distribuição de grandes volumes de produtos em áreas urbanas de grande densidade populacional, caracterizadas por dificuldades de tráfego e estacionamento. Seu principal diferencial em relação ao roteamento clássico é o uso de múltiplos entregadores e a existência de rotas primárias (percorridas pelos veículos) e rotas secundárias (percorridas pelos entregadores). O problema, caracterizado como de Localização-roteamento em dois níveis com janelas de tempo e múltiplos entregadores (2E-LRPTWMD), foi formalmente descrito e formulado por um modelo de programação inteira mista.

Experimentos com exemplos de até 50 clientes, gerados aleatoriamente ou com base em dados reais, indicam que o 2E-LRPTWMD assim como outros problemas de roteamento de interesse, é de difícil resolução, em especial quando as distâncias percorridas por veículos e entregadores são geradas de forma arbitrária (toys e instâncias RC). Nestes casos, o solver de programação matemática empregado enfrentou grandes dificuldades de convergência, produzindo soluções com gaps altos, mesmo após várias horas de processamento.

Perspectivas de pesquisa futura incluem o estudo de formulações alternativas, a extensão do modelo de forma a permitir múltiplas rotas secundárias a partir de um mesmo ponto de parada, e a incorporação de restrições próprias da logística urbana como horários restritos de circulação de veículos pesados em certas regiões das cidades. Outro possível desdobramento do estudo é o desenvolvimento de métodos de solução eficazes, tais como heurísticas e metaheurísticas, para o tratamento de instâncias de porte realista. Nestes casos, o tamanho da instância pode atingir de centenas a milhares de entregas diárias, inviabilizando, portanto, a aplicação de métodos de otimização exatos. 


\section{REFERÊNCIAS BIBLIOGRÁFICAS}

[1] ÁLVAREZ, A.; MUNARI, P. An exact hybrid method for the vehicle routing problem with time windows and multiple deliverymen. Computers \& Operations Research, v. 83, pp. 1-12, 2017.

[2] CLARKE, G.; WRIGHT, J. W. Scheduling of Vehicles from a Central Depot to a Number of Delivery Points. Operations Research, v. 12, n. 4, p. 568-581, 1964.

[3] DE GRANCY, G. S. An adaptive metaheuristic for vehicle routing problems with time windows and multiple service workers. Journal of Universal Computer Science, v. 21, n. 9, p. 1143-1167, 2015.

[4] DE GRANCY, G. S.; REIMANN, M. Vehicle routing problems with time windows and multiple service workers: a systematic comparison between ACO and GRASP. Central European Journal of Operations Research, v. 24, n. 1, p. 29-48, 2016.

[5] MUNARI, P; MORABITO, R. The vehicle routing problem with time windows and multiple deliverymen: exact solution approaches. Relatório técnico. Departamento de Engenharia de Produção, Universidade Federal de São Carlos, 2016. Disponível em: <http://www.optimization-online.org/DB_HTML/2016/01/5289.html>.

[6] PUREZA, V.; MORABITO, R.; REIMANN, M. Vehicle routing with multiple deliverymen: Modeling and heuristic approaches for the VRPTW. European Journal of Operational Research, v. 218, pp. 636-647, 2012.

[7] SOLOMON, M. M.. Algorithms for the vehicle routing and scheduling problems with time window constraints. Operations Research, v. 35, n. 2, p. 254-265, 1987

[8] SOUZA NETO, J. F.; PUREZA, V. Modeling and solving a rich vehicle routing problem for the delivery of goods in urban areas. Pesquisa Operacional, v. 36, n. 3, pp. 421-446, 2016.

[9] UCHOA, E.; PECIN, D.; PESSOA, A.; POGGI, M.; VIDAL, T.; SUBRAMANIAN, A. New benchmark instances for the Capacitated Vehicle Routing Problem. European Journal of Operational Research, v. 257, n. 3, p. 845-858, 2017. 